\title{
Design of PDC Controllers by Matrix Reversibility for Synchronization of Yin and Yang Chaotic Takagi-Sugeno Fuzzy Henon Maps
}

\author{
Chun-Yen Ho, ${ }^{1}$ Hsien-Keng Chen, ${ }^{2}$ and Zheng-Ming Ge ${ }^{1}$ \\ ${ }^{1}$ Department of Mechanical Engineering, National Chiao Tung University, Hsinchu 300, Taiwan \\ ${ }^{2}$ Department of Mechanical Engineering, Hsiuping University of Science and Technology, Dali City, \\ Taichung 412, Taiwan \\ Correspondence should be addressed to Zheng-Ming Ge, zmg@cc.nctu.edu.tw
}

Received 6 June 2012; Revised 8 October 2012; Accepted 9 October 2012

Academic Editor: Zhenya Yan

Copyright (C) 2012 Chun-Yen Ho et al. This is an open access article distributed under the Creative Commons Attribution License, which permits unrestricted use, distribution, and reproduction in any medium, provided the original work is properly cited.

\begin{abstract}
This paper investigates the synchronization of Yin and Yang chaotic T-S fuzzy Henon maps via PDC controllers. Based on the Chinese philosophy, Yin is the decreasing, negative, historical, or feminine principle in nature, while Yang is the increasing, positive, contemporary, or masculine principle in nature. Yin and Yang are two fundamental opposites in Chinese philosophy. The Henon map is an invertible map; so the Henon maps with increasing and decreasing argument can be called the Yang and Yin Henon maps, respectively. Chaos synchronization of Yin and Yang T-S fuzzy Henon maps is achieved by PDC controllers. The design of PDC controllers is based on the linear invertible matrix theory. The T-S fuzzy model of Yin and Yang Henon maps and the design of PDC controllers are novel, and the simulation results show that the approach is effective.
\end{abstract}

\section{Introduction}

Chaotic behavior in nonlinear system is an interesting phenomenon, which is very sensitive in initial conditions. Chaos plays an important role in nonlinear science field [1-4]. In [5], the Yin chaos in continuous time nonlinear system is firstly proposed. In 1990, Pecora and Carroll proposed chaotic synchronization scheme and started a new research interest [6]. In many papers, chaos synchronization of two-dimensional Hênon map system is studied [7-11]. In 1985, Takagi and Sugeno [12] proposed T-S fuzzy model and has been used in complex nonlinear systems successfully [13]. The T-S fuzzy model of the nonlinear systems can exactly be represented as a fuzzy aggregation of some local linear systems. Generally, the implementation of synchronization [14] and control $[15,16]$ in T-S fuzzy model of chaotic systems uses the parallel distributed compensation (PDC) scheme. 
The features of this paper are as follows.

(1) We develop the chaotic Henon map with decreasing argument, which is called Yin chaotic Henon map.

(2) Yin and Yang T-S fuzzy models of chaotic Henon maps are proposed.

(3) The nonlinear discrete time Yin Henon map is the inverse of Yang Henon map. Besides, the design of PDC controllers uses linear invertible matrix theory to achieve chaos synchronization of T-S fuzzy Yin and Yang Henon maps.

This paper is organized as follows. In Section 2, the two-dimensional invertible maps, Yin and Yang chaos are introduced. In Section 3, the Yang and Yin chaos of Henon maps are presented. Section 4, Takagi-Sugeno fuzzy model scheme is given. In Section 5, Yang and Yin T-S fuzzy models of the chaotic Henon maps are given. In Section 6, the design of PDC controllers for Yin and Yang T-S fuzzy synchronization of chaotic Henon maps is given by the linear invertible matrix theory. In Section 7, simulation results are given. In Section 8, conclusions are drawn.

\section{Two-Dimensional Invertible Maps}

Consider the general two-dimensional map form as follows:

$$
\begin{aligned}
& x_{1}\left[n_{1}+1\right]=f_{1}\left\{x_{1}\left[n_{1}\right], x_{2}\left[n_{1}\right]\right\}, \\
& x_{2}\left[n_{1}+1\right]=f_{2}\left\{x_{1}\left[n_{1}\right], x_{2}\left[n_{1}\right]\right\},
\end{aligned}
$$

where $x_{1}\left[n_{1}+1\right], x_{2}\left[n_{1}+1\right]$ are the functions of $x_{1}\left[n_{1}\right], x_{2}\left[n_{1}\right], n_{1}$ is the positive argument, that is, $n_{1}=0,1,2,3, \ldots, n$. The map is invertible [17] if (2.1) can be solved uniquely for $x_{1}\left[n_{1}\right]$ and $x_{2}\left[n_{1}\right]$ as functions of $x_{1}\left[n_{1}+1\right]$ and $x_{2}\left[n_{1}+1\right]$. The inversed map system can be written from (2.1):

$$
\begin{aligned}
& \widehat{x}_{1}\left[n_{2}-1\right]=g_{1}\left\{\widehat{x}_{1}\left[n_{2}\right], \widehat{x}_{2}\left[n_{2}\right]\right\}, \\
& \widehat{x}_{2}\left[n_{2}-1\right]=g_{2}\left\{\widehat{x}_{1}\left[n_{2}\right], \widehat{x}_{2}\left[n_{2}\right]\right\},
\end{aligned}
$$

where $n_{2}$ is the negative argument, that is, $n_{2}=0,-1,-2,-3, \ldots,-n$. The chaos obtained for negative argument is called Yin chaos, while that obtained for positive argument is called Yang chaos.

\section{The Yang Chaos of Henon Map System and Yin Chaos of Inversed Henon Map System}

Consider the Henon map system [18]:

$$
\begin{gathered}
x_{1}\left[n_{1}+1\right]=-a_{1} x_{1}^{2}\left[n_{1}\right]+x_{2}\left[n_{1}\right]+1, \\
x_{2}\left[n_{1}+1\right]=b_{1} x_{1}\left[n_{1}\right],
\end{gathered}
$$




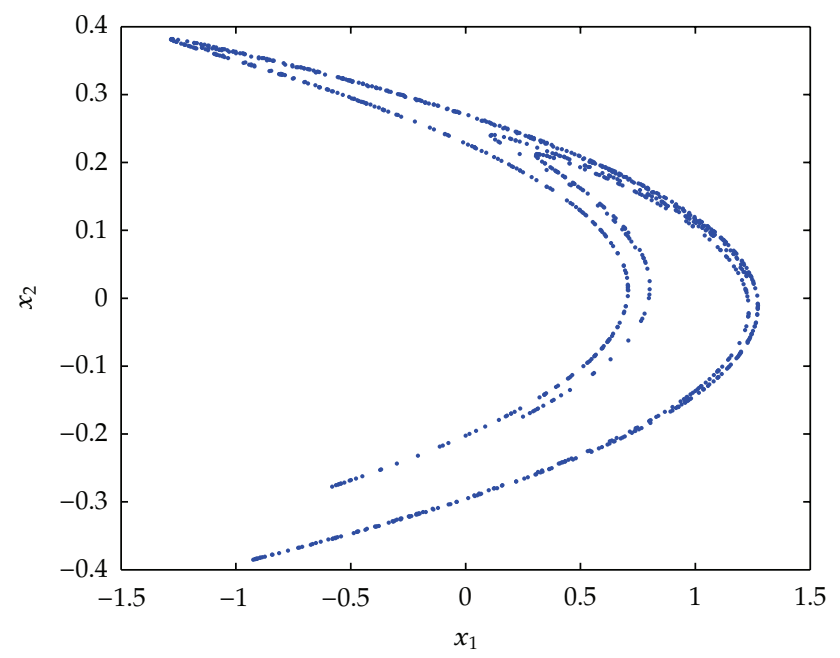

Figure 1: Phase portrait of the chaotic Yang Henon map system with $a_{1}=1.4, b_{1}=0.3$.

where $a_{1}=1.4, b_{1}=0.3$ and positive argument $n_{1}=0,1,2,3, \ldots, n$. The Yang chaotic behaviors of Henon map system are quoted [19] by phase portraits in Figure 1.

The Henon map system is invertible if $b_{1} \neq 0$. The inversed Henon map system can be described as follows:

$$
\begin{gathered}
\widehat{x}_{1}\left[n_{2}-1\right]=\frac{1}{b_{1}} \widehat{x}_{2}\left[n_{2}\right], \\
\widehat{x}_{2}\left[n_{2}-1\right]=\widehat{x}_{1}\left[n_{2}\right]+\frac{a_{1}}{b_{1}^{2}} \widehat{x}_{2}^{2}\left[n_{2}\right]-1 .
\end{gathered}
$$

The parameters $1 / b_{1}, a_{1} / b_{1}^{2}$ are replaced by $b_{2}, a_{2}$, respectively, where $b_{2}=0.3, a_{2}=1.4$ and negative argument $n_{2}=-1,-2,-3, \ldots,-n$. The Yin chaotic behaviors of inversed Henon map system are shown in Figure 2.

Comparing Figure 1 and Figure 2, it is surprisingly noted that Figure 2 gives new information for famous Henon map system. Since 1976 [18], traditional studies of Henon system have only been devoted to its behaviors with positive argument. Now it is discovered that with negative argument or negative time, a new continent is waiting for us in the future study of either nonlinear map systems or nonlinear continuous systems.

\section{Takagi-Sugeno Fuzzy Model}

The T-S fuzzy model [12] can represent a nonlinear system by linear state space which is described by fuzzy IF-THEN rules.

Consider the following discrete time T-S fuzzy rules:

$$
\begin{gathered}
R^{i}: \operatorname{IF} p_{1}(n) \text { is } M_{i 1}, \ldots, \text { and } p_{q}(n) \text { is } M_{i q}, \\
\operatorname{THEN} x(n+1)=A_{i} x(n)+c,
\end{gathered}
$$




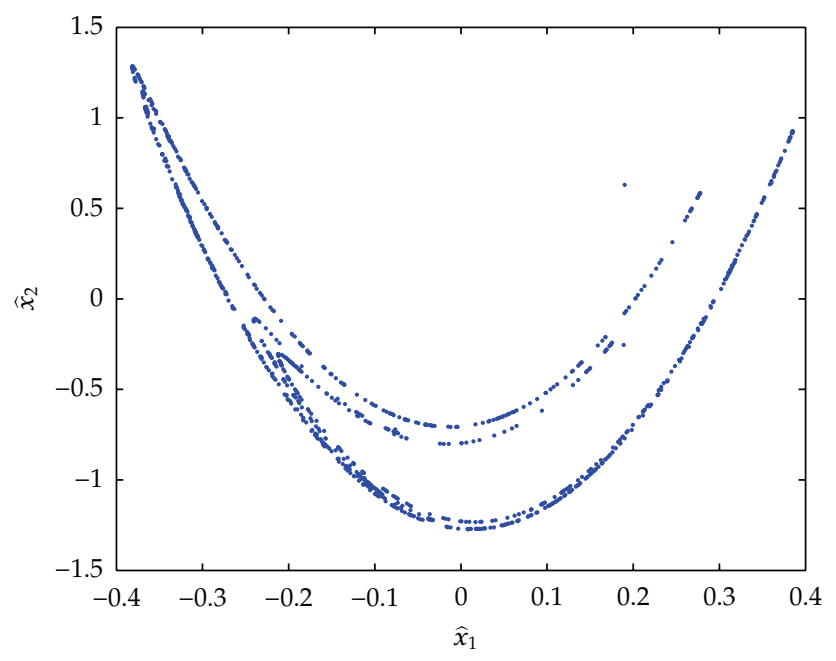

Figure 2: Phase portrait of the chaotic Yin Henon map system with $a_{2}=1.4, b_{2}=0.3$.

where $i=1,2, \ldots, r$ ( $r$ is the number of fuzzy rules), $x(n) \in R^{j}$ represents the state vector, $A_{i} \in R^{j \times j}$ are known matrix, $p_{1}(n), p_{2}(n), \ldots, p_{q}(n)$ are premise variables, $M_{i h}$ is a fuzzy set $(h=1,2, \ldots, q)$, and $c \in R^{j}$ is a constant vector. The final discrete time T-S fuzzy system is inferred as follows:

$$
x(n+1)=\frac{\sum_{i=1}^{r} \omega_{i}(p(n))\left(A_{i} x(n)+c\right)}{\sum_{i=1}^{r} \omega_{i}(p(n))},
$$

where

$$
\omega_{i}(p(n))=\prod_{h=1}^{q} M_{i h}\left(p_{h}(n)\right)
$$

in which $M_{i h}\left(p_{h}(n)\right)$ is the degree of membership of $p_{h}(n)$ in $M_{i h}$. The following conditions must be satisfied:

$$
\begin{aligned}
& \sum_{i=1}^{r} \omega_{i}(p(n))>0, \quad i=1,2, \ldots, r . \\
& \omega_{i}(p(n)) \geq 0,
\end{aligned}
$$

let $\mu_{i}(p(n))=\omega_{i}(p(n)) / \sum_{i=1}^{r} \omega_{i}(p(n)),(4.2)$ can be rewritten as

$$
x(n+1)=\sum_{i=1}^{r} \mu_{i}(p(n))\left(A_{i} x(n)+c\right) .
$$


Note that

$$
\begin{aligned}
& \sum_{i=1}^{r} \mu_{i}(p(n))=1, \quad i=1,2, \ldots, r . \\
& \mu_{i}(p(n)) \geq 0 .
\end{aligned}
$$

\section{Yang and Yin T-S Fuzzy Models of the Chaotic Henon Maps}

Figures 1 and 2 show that the ranges of the states $x_{1}(n)$ and $\hat{x}_{2}(n)$ for Yang and Henon map are from -1.4 to $1.4\left(x_{1}(n), \widehat{x}_{2} \in[-1.4,1.4]\right)$, respectively. The Yang and Yin T-S fuzzy models of chaotic Henon map systems can be obtained as follows.

Yang T-S fuzzy model of chaotic Henon map system:

$$
\begin{aligned}
& R^{1}: \text { IF } x_{1}\left(n_{1}\right) \text { is } M_{11} \text {, THEN } x\left(n_{1}+1\right)=A_{1} x\left(n_{1}\right)+c, \\
& R^{2}: \text { IF } x_{1}\left(n_{1}\right) \text { is } M_{21} \text {, THEN } x\left(n_{1}+1\right)=A_{2} x\left(n_{1}\right)+c .
\end{aligned}
$$

Yin T-S fuzzy model of chaotic Henon map system:

$$
\begin{aligned}
& R^{1}: \operatorname{IF} \widehat{x}_{2}\left(n_{2}\right) \text { is } \widehat{M}_{11}, \operatorname{THEN} \widehat{x}\left(n_{2}-1\right)=\widehat{A}_{1} \widehat{x}\left(n_{2}\right)+\widehat{c}, \\
& R^{2}: \operatorname{IF} \widehat{x}_{2}\left(n_{2}\right) \text { is } \widehat{M}_{21} \text {, THEN } \widehat{x}\left(n_{2}-1\right)=\widehat{A}_{2} \widehat{x}\left(n_{2}\right)+\widehat{c} .
\end{aligned}
$$

In (5.1), $x\left(n_{1}\right)=\left(x_{1}\left(n_{1}\right), x_{2}\left(n_{1}\right)\right)^{T}, n_{1}=0,1,2,3, \ldots, n, A_{1}=\left[\begin{array}{cc}-a_{1}(1.4) & 1 \\ b_{1} & 0\end{array}\right]$, and $A_{2}=\left[\begin{array}{rr}-a_{1}(-1.4) & 1 \\ b_{1} & 0\end{array}\right]$, $c=(1,0)^{T}$.

The membership functions are chosen as follows:

$$
\begin{aligned}
& M_{11}\left(x_{1}\left(n_{1}\right)\right)=\frac{1}{2}\left(1+\frac{x_{1}\left(n_{1}\right)}{1.4}\right) \\
& M_{21}\left(x_{1}\left(n_{1}\right)\right)=\frac{1}{2}\left(1-\frac{x_{1}\left(n_{1}\right)}{1.4}\right) .
\end{aligned}
$$

In (5.2), $\widehat{x}\left(n_{2}\right)=\left(\widehat{x}_{1}\left(n_{2}\right), \widehat{x}_{2}\left(n_{2}\right)\right)^{T}, n_{2}=0,-1,-2,-3, \ldots,-n, \widehat{A}_{1}=\left[\begin{array}{cc}0 & b_{2} \\ 1 & a_{2}(1.4)\end{array}\right], \widehat{A}_{2}=\left[\begin{array}{cc}0 & b_{2} \\ 1 & a_{2}(-1.4)\end{array}\right]$, and $\widehat{c}=(0,-1)^{T}$.

The membership functions are chosen as follows:

$$
\begin{aligned}
& \widehat{M}_{11}\left(\widehat{x}_{2}\left(n_{2}\right)\right)=\frac{1}{2}\left(1+\frac{\widehat{x}_{2}\left(n_{2}\right)}{1.4}\right) \\
& \widehat{M}_{21}\left(\widehat{x}_{2}\left(n_{2}\right)\right)=\frac{1}{2}\left(1-\frac{\widehat{x}_{2}\left(n_{2}\right)}{1.4}\right) .
\end{aligned}
$$




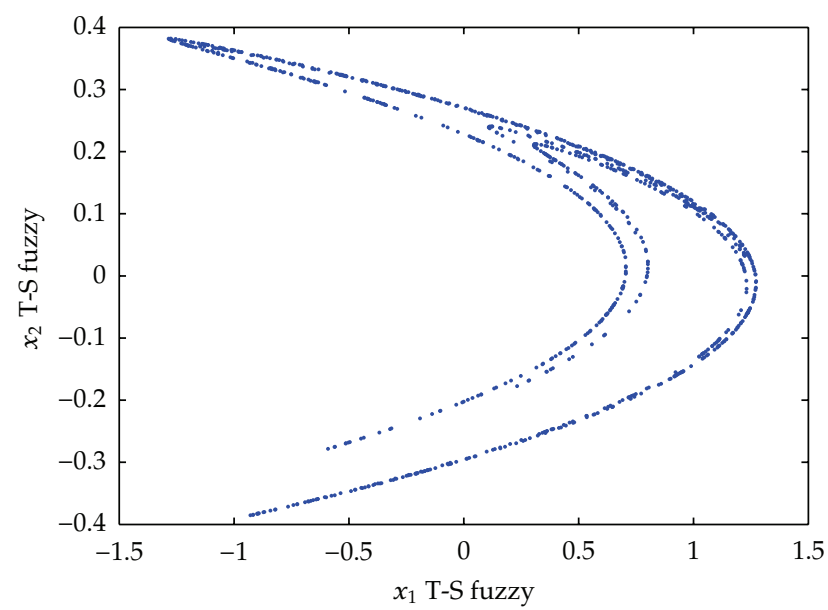

Figure 3: Phase portrait of Yang T-S fuzzy model of Henon map system with $a_{1}=1.4, b_{1}=0.3$.

Then, applying the product-inference rule, singleton fuzzifier, and the center of gravity defuzzifier to the above fuzzy rule base ((5.1) and (5.2)), the overall Yang and Yin fuzzy chaotic maps can be shown in (5.5) and (5.6), respectively

$$
\begin{aligned}
& x\left(n_{1}+1\right)=\sum_{i=1}^{2} \mu_{i}\left(x_{1}\left(n_{1}\right)\right)\left(A_{i} x\left(n_{1}\right)+c\right), \\
& \widehat{x}\left(n_{2}+1\right)=\sum_{i=1}^{2} \mu_{i}\left(\widehat{x}_{2}\left(n_{2}\right)\right)\left(A_{i} \widehat{x}\left(n_{2}\right)+\widehat{c}\right),
\end{aligned}
$$

where

$$
\begin{aligned}
& \mu_{i}\left(x_{1}\left(n_{1}\right)\right)=\frac{M_{i 1}\left(x_{1}\left(n_{1}\right)\right)}{\sum_{i=1}^{2}\left(M_{i 1}\left(x_{1}\left(n_{1}\right)\right)\right)}, \\
& \mu_{i}\left(\widehat{x}_{2}\left(n_{2}\right)\right)=\frac{M_{i 1}\left(\widehat{x}_{2}\left(n_{2}\right)\right)}{\sum_{i=1}^{2}\left(M_{i 1}\left(\widehat{x}_{2}\left(n_{2}\right)\right)\right)} .
\end{aligned}
$$

The Yang T-S fuzzy model of chaotic Henon map system is given in Figure 3. It is clear to see that the derived Yang T-S fuzzy model is equivalent to the original chaotic map in Figure 1. Otherwise, the Yin T-S fuzzy model of chaotic Henon map system is given in Figure 4 and is equivalent to the original chaotic map in Figure 2.

\section{Controller Design for Chaos Synchronization of Yin and Yang T-S Fuzzy Model of Henon Maps}

Let Yang T-S fuzzy chaotic map (5.5) be as the drive system and the response system as follows:

$$
y(n+1)=\sum_{i=1}^{2} \mu_{i}\left(y_{1}(n)\right)\left(A_{i} y(n)+c\right)+u(n)
$$




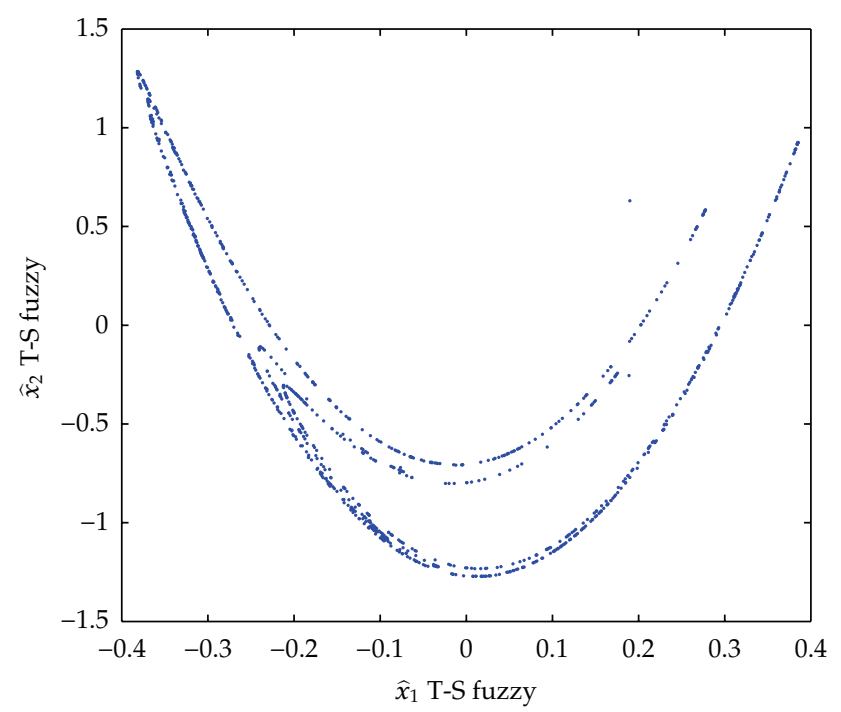

Figure 4: Phase portrait of Yin T-S fuzzy model of Henon map system with $a_{2}=1.4, b_{2}=0.3$.

The target for the design of a fuzzy controller $u(n)$ is synchronized the two discrete time chaotic maps by the PDC technique as follows:

$$
u(n)=\sum_{i=1}^{2} \mu_{i}\left(x_{1}(n)\right) K_{i} x(n)-\sum_{i=1}^{2} \mu_{i}\left(y_{1}(n)\right) K_{i} y(n) .
$$

Define the error dynamics:

$$
e(n)=x(n)-y(n)
$$

From (6.1) and (6.2), the closed loop synchronization error dynamics (6.3) is arranged as

$$
e(n+1)=\sum_{i=1}^{2} \mu_{i}\left(x_{1}(n)\right)\left(A_{i}+K_{i}\right) x(n)-\sum_{i=1}^{2} \mu_{i}\left(y_{1}(n)\right)\left(A_{i}+K_{i}\right) y(n) .
$$

According to stability theory for discrete linear system, it is known that a matrix $H$ if and only if the eigenvalues of matrix $H$ are less than 1 in absolute value [20], the error dynamics (6.3) is asymptotically stable, in other words, the chaos synchronization is achieved. Let a Schur stable matrix $H_{1}$ for Yang controller design satisfy the following equation:

$$
A_{1}+K_{1}=A_{2}-K_{2}=H_{1} .
$$


Since the system is the T-S fuzzy model of invertible map and the determinants of $K_{1}, K_{2}$ are not equal to zero, the Yin fuzzy controllers meet the following equation:

$$
\widehat{K}_{1}=\widehat{K}_{1}^{-1}, \quad \widehat{K}_{2}=\widehat{K}_{2}^{-1} .
$$

So the Schur matrix $\mathrm{H}_{2}$ for Yin controller design satisfy the following equation:

$$
A_{1}^{-1}+K_{1}^{-1}=A_{2}^{-1}-K_{2}^{-1}=H_{2}
$$

We need to find the $K_{1}, K_{2}, \widehat{K}_{1}, \widehat{K}_{2}$ to meet (6.5) and (6.7), that is, if all the eigenvalues of $H_{1}, H_{2}$ are less than 1 in absolute value, the Yang and Yin T-S fuzzy chaotic Henon maps are synchronized individually. Because (6.7) is not the invertible of (6.5) (the invertible of (6.5) is $\left.\left(A_{1}+K_{1}\right)^{-1}=\left(A_{2}-K_{2}\right)^{-1}=\left(H_{1}\right)^{-1}\right)$, we can find $H_{1}$ and $H_{2}$ to meet Schur matrix.

\section{Simulation Results}

In order to apply the proposed Yin-Yang chaos synchronization scheme by PDC technique, the T-S fuzzy model is used. Yang T-S fuzzy model of Henon map system as drive system is described in (5.1), (5.3), (5.5), and (5.7). Yang T-S fuzzy model of Henon map system as response system is described by (6.1) and (6.2):

$$
y\left(n_{1}+1\right)=\sum_{i=1}^{2} \mu_{i}\left(y_{1}\left(n_{1}\right)\right)\left(A_{i} y\left(n_{1}\right)+c\right)+u\left(n_{1}\right)
$$

where

$$
\begin{gathered}
A_{1}=\left[\begin{array}{cc}
-a_{1}(1.4) & 1 \\
b_{1} & 0
\end{array}\right], \quad A_{2}=\left[\begin{array}{cc}
-a_{1}(-1.4) & 1 \\
b_{1} & 0
\end{array}\right], \quad c=[1,0]^{T}, \\
u(n)=\sum_{i=1}^{2} \mu_{i}\left(x_{1}\left(n_{1}\right)\right) K_{i} x\left(n_{1}\right)-\sum_{i=1}^{2} \mu_{i}\left(y_{1}\left(n_{1}\right)\right) K_{i} y\left(n_{1}\right) .
\end{gathered}
$$

According to the proposed Yin-Yang chaos synchronization by PDC technique, the stable matrix $H_{1}$ is chosen as $H_{1}=\left[\begin{array}{cc}-0.96 & 0.5 \\ -0.7 & 0\end{array}\right]$, where the two eigenvalues of $H_{1}$ are $-0.48,-0.48$. The Yang fuzzy controller can be obtained by (6.5):

$$
\begin{aligned}
& K_{1}=\left[\begin{array}{cc}
1 & -0.5 \\
-1 & 0
\end{array}\right], \\
& K_{2}=\left[\begin{array}{cc}
2.92 & 0.5 \\
1 & 0
\end{array}\right] .
\end{aligned}
$$

Yang T-S fuzzy model of chaotic Henon maps as drive and response system is simulated with initial conditions $\left[x_{1}(0) x_{2}(0)\right]^{T}=\left[\begin{array}{ll}0.63 & 0.19\end{array}\right]^{T},\left[y_{1}(0) y_{2}(0)\right]^{T}=\left[\begin{array}{ll}0.4 & 0.1\end{array}\right]^{T}$. 


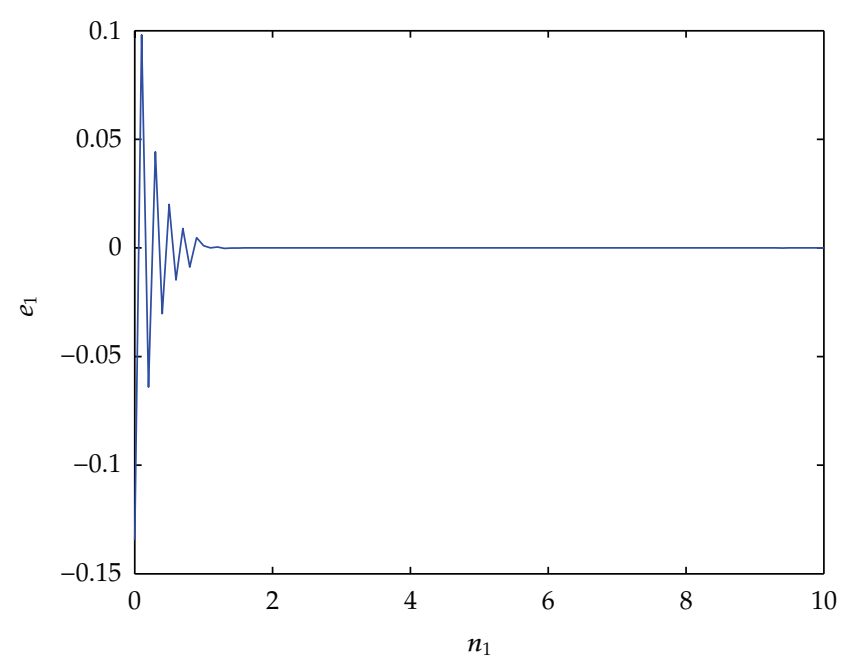

Figure 5: Error dynamics $e_{1}$ for chaos synchronization of Yang T-S fuzzy model of Henon map system.

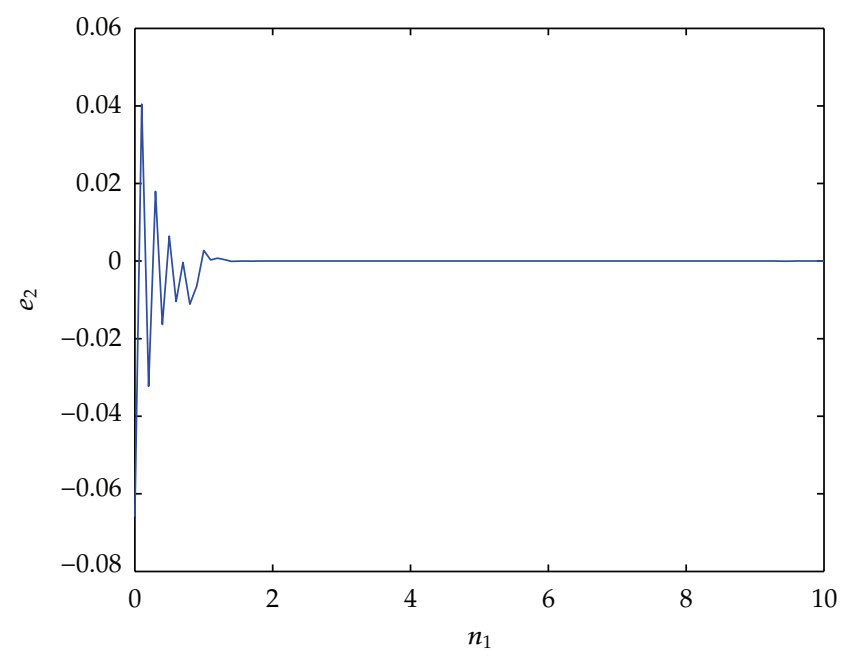

Figure 6: Error dynamics $e_{2}$ for chaos synchronization of Yang T-S fuzzy model of Henon map system.

Simulation results show that the error dynamics approach to be asymptotically stable in Figures 5 and 6. The Yin fuzzy controllers can be obtained by (6.6):

$$
\begin{aligned}
& \widehat{K}_{1}=K_{1}^{-1}=\left[\begin{array}{cc}
0 & -1 \\
-2 & -2
\end{array}\right] \\
& \widehat{K}_{2}=K_{2}^{-1}=\left[\begin{array}{cc}
0 & 1 \\
2 & -5.84
\end{array}\right] .
\end{aligned}
$$

From (6.7), we can obtain the Schur matrix $H_{2}=\left[\begin{array}{cc}0 & -0.7 \\ -1 & -0.04\end{array}\right]$, where the initial conditions $\left[\hat{x}_{1}(0) \hat{x}_{2}(0)\right]^{T}=\left[\begin{array}{ll}0.19 & 0.63\end{array}\right]^{T},\left[\hat{y}_{1}(0) \hat{y}_{2}(0)\right]^{T}=\left[\begin{array}{ll}0.1 & 0.4\end{array}\right]^{T}$ and the two eigenvalues of $H_{2}$ are 0.817, 


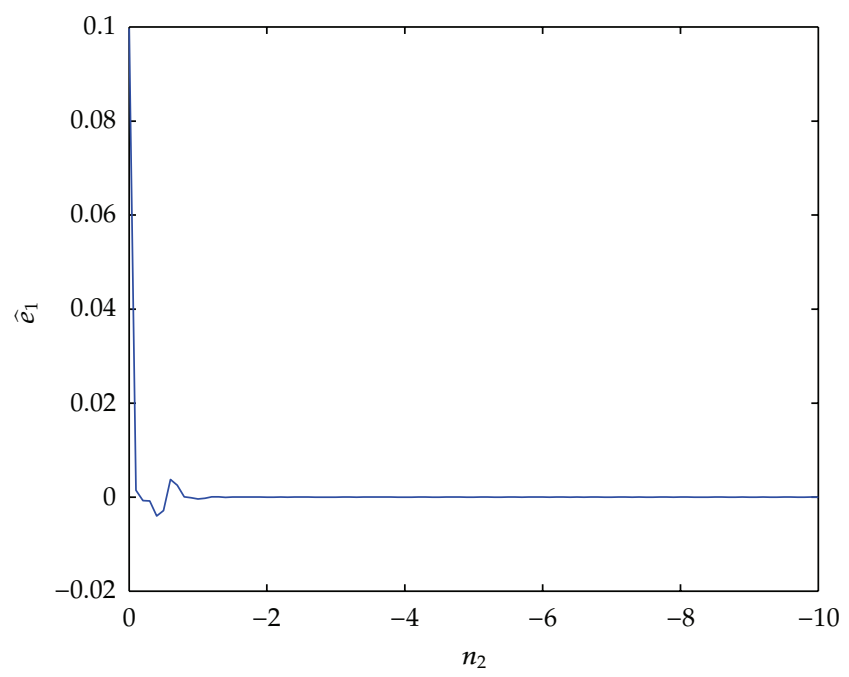

Figure 7: Error dynamics $\widehat{e}_{1}$ for chaos synchronization of Yin T-S fuzzy model of Henon map system.

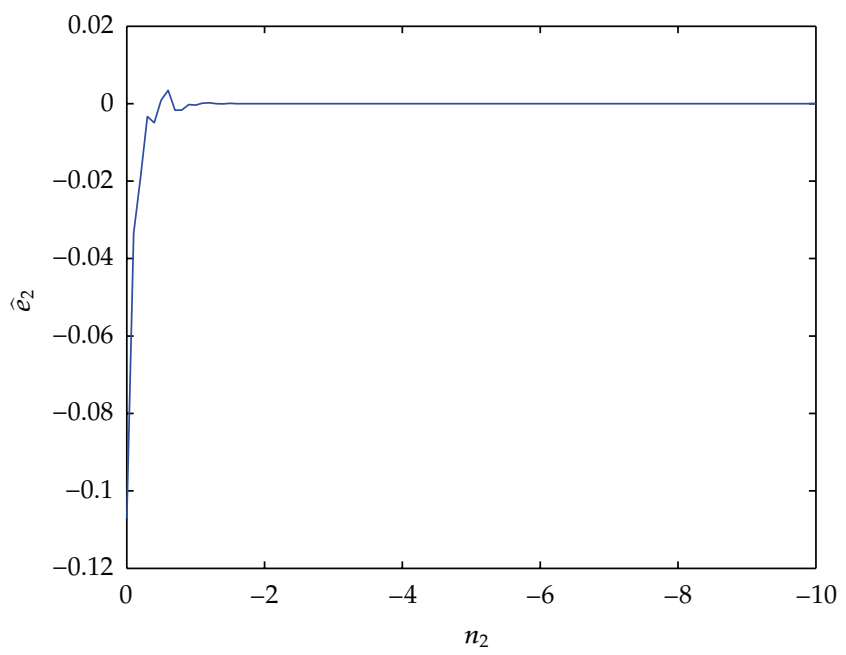

Figure 8: Error dynamics $\widehat{e}_{2}$ for chaos synchronization of Yin T-S fuzzy model of Henon map system.

-0.857 . Simulation results show that the error dynamics approach to be asymptotically stable in Figures 7 and 8. In other words, chaos synchronization is achieved.

\section{Conclusions}

Yin chaos of inversed Henon map system and the Yin T-S fuzzy model of chaotic Henon map system are proposed. The T-S fuzzy model of a chaotic system can exactly be represented as a fuzzy aggregation of some local linear systems. As a result, the conventional linear system theory can be applied. Since nonlinear discrete time Henon map is an invertible map, we develop the Yang and Yin T-S fuzzy model of Henon map system. The design of the Yin fuzzy controller is fleetly obtained by the inverse of Yang fuzzy controller, the concern is that the Yin 
chaotic system must be an inverse of the Yang chaotic system and meet the stability theory for discrete linear system. In secure communication, the chaotic system as transmitter and the inverse chaotic system as receiver are hard to achieve due to the nonlinearity; however the Yin and Yang T-S fuzzy models of Henon map system can overcome these difficulties.

\section{Acknowledgments}

This research was partially supported by the National Science Council, China, under Grant no. NSC 101-2221-E164-008. The authors would like to thank the anonymous reviewers for their insightful comments and suggestions in this paper.

\section{References}

[1] H. N. Agiza, “Chaos synchronization of Lü dynamical system,” Nonlinear Analysis: Theory, Methods $\mathcal{E}$ Applications, vol. 58, no. 1-2, pp. 11-20, 2004.

[2] E. E. Escultura, "Dynamic modeling of chaos and turbulence," Nonlinear Analysis: Theory, Methods and Applications, vol. 63, no. 5-7, pp. e519-e532, 2005.

[3] A. H. Nayfeh and B. Balachandran, Applied Nonlinear Dynamics, John Wiley \& Sons, New York, NY, USA, 1995.

[4] F. Takeo, "Chaos and hypercyclicity for solution semigroups to some partial differential equations," Nonlinear Analysis: Theory, Methods and Applications, vol. 63, no. 5-7, pp. e1943-e1953, 2005.

[5] Z.-M. Ge and S.-Y. Li, "Yang and Yin parameters in the Lorenz system," Nonlinear Dynamics, vol. 62, no. 1-2, pp. 105-117, 2010.

[6] L. M. Pecora and T. L. Carroll, "Synchronization in chaotic systems," Physical Review Letters, vol. 64, no. 8, pp. 821-824, 1990.

[7] X. Huang, P. Jia, B. Liu, and L. Wang, "Chaotic particle swarm optimization for synchronization of finite dimensional Hénon dynamical system," in Proceedings of the 6th International Conference on Natural Computation (ICNC '10), vol. 5, pp. 2600-2604, August 2010.

[8] D. Arroyo, G. Alvarez, S. Li, C. Li, and J. Nunez, "Cryptanalysis of a discrete-time synchronous chaotic encryption system," Physics Letters A, vol. 372, no. 7, pp. 1034-1039, 2008.

[9] Y. Xue and S. Yang, "Synchronization of discrete-time spatiotemporal chaos via adaptive fuzzy control," Chaos, Solitons and Fractals, vol. 17, no. 5, pp. 967-973, 2003.

[10] B. Cazelles, G. Boudjema, and N. P. Chau, "Adaptive synchronization of globally coupled chaotic oscillators using control in noisy environments," Physica D: Nonlinear Phenomena, vol. 103, no. 1-4, pp. 452-465, 1997.

[11] C.-S. Zhou and T.-L. Chen, "Robust communication via chaotic synchronization based on contraction maps," Physics Letters A, vol. 225, no. 1-3, pp. 60-66, 1997.

[12] T. Takagi and M. Sugeno, "Fuzzy identification of systems and its applications to modeling and control," IEEE Transactions on Systems, Man and Cybernetics, vol. 15, no. 1, pp. 116-132, 1985.

[13] H. Han, C. Y. Su, and S. Murakami, "Adaptive fuzzy control for a class of discrete-time nonlinear systems," in Proceedings of the 10th IEEE International Conference on Fuzzy Systems, vol. 2, pp. 892-895, December 2001.

[14] K. Y. Lian, C. S. Chiu, T. S. Chiang, and P. Liu, "LMI-based fuzzy chaotic synchronization and communications," IEEE Transactions on Fuzzy Systems, vol. 9, no. 4, pp. 539-553, 2001.

[15] K. Tanaka, T. Ikeda, and H. O. Wang, "A unified approach to controlling chaos via an LMI-based fuzzy control system design," IEEE Transactions on Circuits and Systems I, vol. 45, no. 10, pp. 1021-1040, 1998.

[16] Y.-Y. Hou, "Controlling chaos in permanent magnet synchronous motor control system via fuzzy guaranteed cost controller," Abstract and Applied Analysis, vol. 2012, Article ID 650863, 10 pages, 2012.

[17] E. Ott, "Strange attractors and chaotic motions of dynamical systems," Reviews of Modern Physics, vol. 53, no. 4, pp. 655-671, 1981.

[18] M. Hénon, "A two-dimensional mapping with a strange attractor," Communications in Mathematical Physics, vol. 50, no. 1, pp. 69-77, 1976.

[19] S. Lynch, Dynamical Systems with Applications Using MATLAB, Birkhäuser, Basel, Switzerland, 2004.

[20] A. J. Lichtenberg and M. A. Lieberman, Regular and Chaotic Dynamics, vol. 38, Springer, New York, NY, USA, 2nd edition, 1992. 


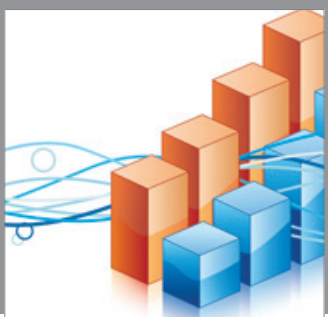

Advances in

Operations Research

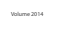

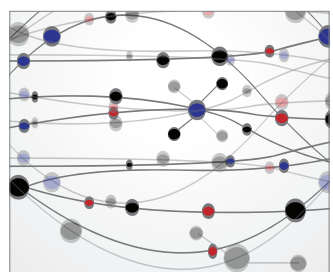

\section{The Scientific} World Journal
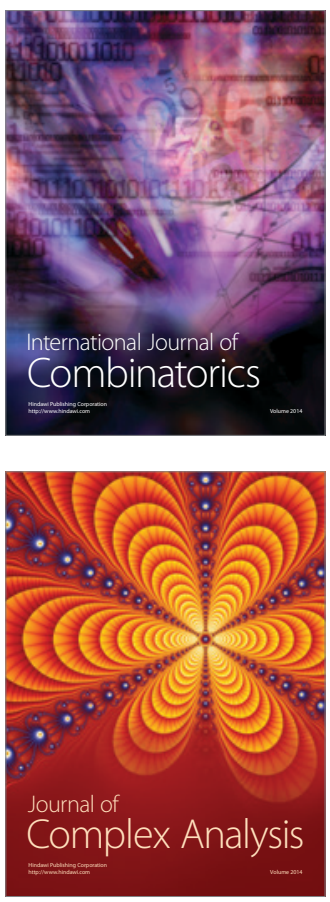

International Journal of

Mathematics and

Mathematical

Sciences
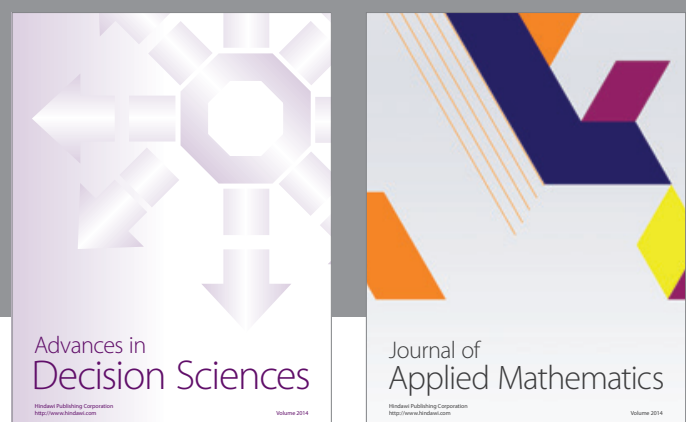

Journal of

Applied Mathematics
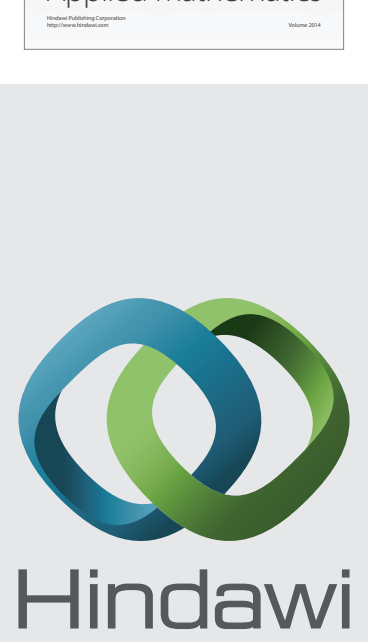

Submit your manuscripts at http://www.hindawi.com
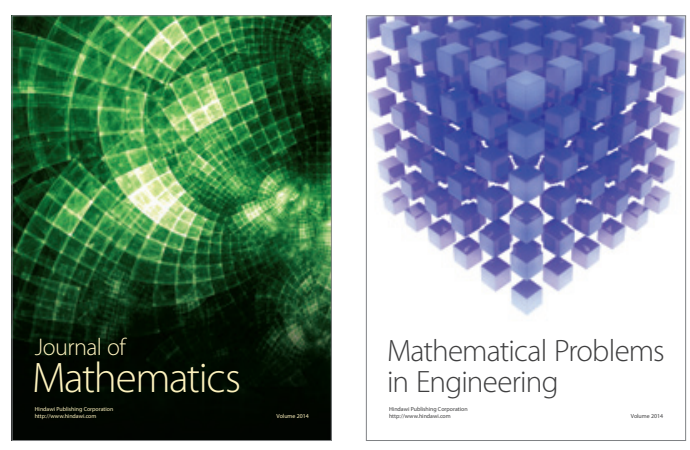

Mathematical Problems in Engineering
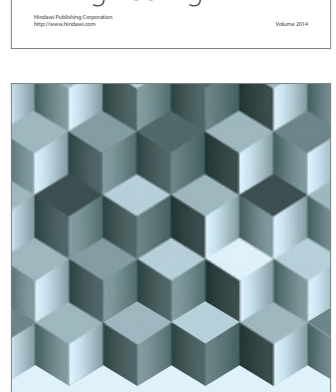

Journal of

Function Spaces
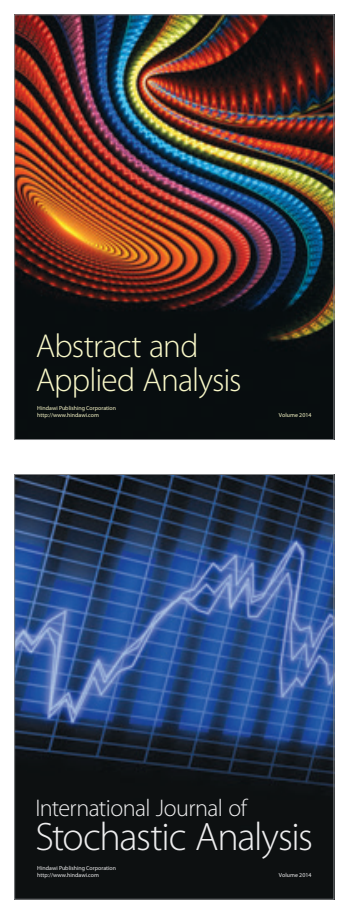

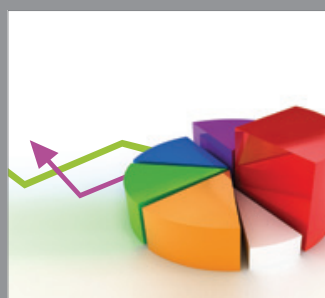

ournal of

Probability and Statistics

Promensencen
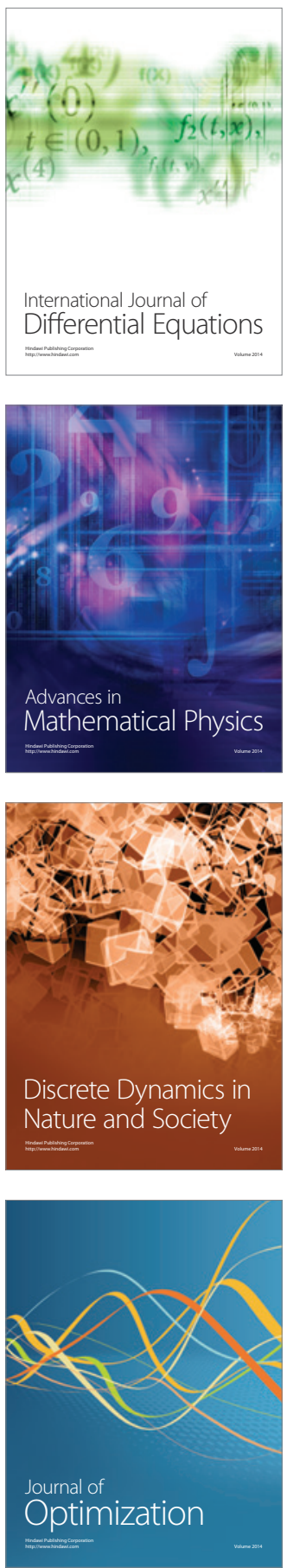